This paper is available online at http://www.tjm.nsysu.edu.tw/

\title{
A $q$-EXTENSION OF THE ERKUS-SRIVASTAVA POLYNOMIALS IN SEVERAL VARIABLES
}

\author{
Esra Erkuş-Duman
}

\begin{abstract}
Recently, Erkus and Srivastava [Integral Transform. Spec. Funct. 174 (2006), 267-273] have introduced and systematically investigated a unified presentation of some families of multivariable polynomials. In this paper, we study a basic (or $q-$ ) analogue of these polynomials, which we construct here.
\end{abstract}

\section{INTRODUCTION}

Recently, Erkus and Srivastava [3] have introduced and systematically investigated a general family of multivariable polynomials generated by

$$
\prod_{j=1}^{r}\left\{\left(1-x_{j} t^{m_{j}}\right)^{-\alpha_{j}}\right\}=\sum_{n=0}^{\infty} u_{n}^{\left(\alpha_{1}, \ldots, \alpha_{r}\right)}\left(x_{1}, \ldots, x_{r}\right) t^{n}
$$

where $\alpha_{j} \in \mathbb{C},(j=1, \ldots, r),|t|<\min \left\{\left|x_{1}\right|^{-1 / m_{1}}, \ldots,\left|x_{r}\right|^{-1 / m_{r}}\right\}$. Clearly, (1.1) yields the following explicit representation

$$
u_{n}^{\left(\alpha_{1}, \ldots, \alpha_{r}\right)}\left(x_{1}, \ldots, x_{r}\right)=\sum_{m_{1} k_{1}+\ldots+m_{r} k_{r}=n}\left(\alpha_{1}\right)_{k_{1}} \ldots\left(\alpha_{r}\right)_{k_{r}} \frac{x_{1}^{k_{1}}}{k_{1} !} \ldots \frac{x_{r}^{k_{r}}}{k_{r} !}
$$

where $(\lambda)_{k}:=\Gamma(\lambda+k) / \Gamma(\lambda)$ denotes the Pochhammer symbol.

In this paper, we first introduce a basic (or $q-$ ) analogue of the generalized multivariable polynomials by (1.2). So we need some definitions and notations from the $q$-analysis. For detailed explanations, see, for example, Jain and Srivastava [4].

Received April 10, 2006, accepted August 9, 2006.

Communicated by H. M. Srivastava.

2000 Mathematics Subject Classification: Primary 33C45, 33D50.

Key words and phrases: Generating function, Addition formula, Lagrange polynomials, LagrangeHermite polynomials, Chan-Chyan-Srivastava polynomials. 
For a real or complex number $q(|q|<1)$, put

$$
(\lambda ; q)_{\infty}=\prod_{j=0}^{\infty}\left(1-\lambda q^{j}\right)
$$

let $(\lambda ; q)_{\mu}$ be defined by

$$
(\lambda ; q)_{\mu}:=\frac{(\lambda ; q)_{\infty}}{\left(\lambda q^{\mu} ; q\right)_{\infty}},
$$

for arbitrary parameters $\lambda$ and $\mu$, so that if $n=1,2, \ldots$ then, $(\lambda ; q)_{n}:=(1-\lambda)(1-$ $\lambda q) \ldots\left(1-\lambda q^{n-1}\right)$; and also if $n=0$, then $(\lambda ; q)_{0}:=1$ (see, for instance, [7]).

\section{Construction of the Polynomials}

We construct a basic (or $q-$ ) analogue of the generalized multivariable polynomials $u_{n, q}^{\left(\alpha_{1}, \ldots, \alpha_{r}\right)}\left(x_{1}, \ldots, x_{r}\right)$ given by (1.2), generated by

$$
\prod_{i=1}^{r} \frac{1}{\left(x_{i} t^{m_{i}} ; q\right)_{\alpha_{i}}}=\sum_{n=0}^{\infty} u_{n, q}^{\left(\alpha_{1}, \ldots, \alpha_{r}\right)}\left(x_{1}, \ldots, x_{r}\right) t^{n},
$$

where $|t|<\min \left\{\left|x_{1}\right|^{-1 / m_{1}}, \ldots,\left|x_{r}\right|^{-1 / m_{r}}\right\}$.

Using the well-known $q$-binomial theorem (see, for instance, [6, p. 241-248], [7, p. 138]) and (1.3) we get

$$
\frac{1}{\left(x_{i} t^{m_{i}} ; q\right)_{\alpha_{i}}}=\sum_{k=0}^{\infty} \frac{\left(q^{\alpha_{i}}, q\right)_{k}}{(q, q)_{k}}\left(x_{i} t^{m_{i}}\right)^{k} ; \quad i=1, \ldots, r .
$$

Now applying the well-known equality for double series

$$
\sum_{n=0}^{\infty} \sum_{k=0}^{\infty} A(k, n)=\sum_{n=0}^{\infty} \sum_{k=0}^{n} A(k, n-k)
$$

(see [5]) we conclude that

$$
\begin{aligned}
\prod_{i=1}^{r} \frac{1}{\left(x_{i} t^{m_{i}} ; q\right)_{\alpha_{i}}}= & \sum_{n=0}^{\infty}\left\{\sum_{m_{1} k_{1}+\ldots+m_{r} k_{r}=n}\left(q^{\alpha_{k_{1}}}, q\right)_{k_{1}} \ldots\left(q^{\alpha_{k_{r}}}, q\right)_{k_{r}}\right. \\
& \left.\times \frac{x_{1}^{k_{1}}}{(q, q)_{k_{1}}} \ldots \frac{x_{r}^{k_{r}}}{(q, q)_{k_{r}}}\right\} t^{n} .
\end{aligned}
$$

By (2.1) and (2.2) a basic (or $q-$ ) analogue of the generalized multivariable polynomials are 


$$
\begin{aligned}
& u_{n, q}^{\left(\alpha_{1}, \ldots, \alpha_{r}\right)}\left(x_{1}, \ldots, x_{r}\right) \\
= & \sum_{m_{1} k_{1}+\ldots+m_{r} k_{r}=n}\left(q^{\alpha_{1}}, q\right)_{k_{1}} \ldots\left(q^{\alpha_{k_{r}}}, q\right)_{k_{r}} \frac{x_{1}^{k_{1}}}{(q, q)_{k_{1}}} \ldots \frac{x_{r}^{k_{r}}}{(q, q)_{k_{r}}} .
\end{aligned}
$$

\section{Bilinear and Bilateral Generating Functions}

In this section we derive several families of bilinear and bilateral generating functions for the basic (or $q-$ ) analogue of the generalized multivariable polynomials given by $(2.3)$.

Theorem 3.1. Corresponding to an identically non-vanishing function $\Omega_{\mu}\left(y_{1}, \ldots, y_{s}\right)$ of complex variables $y_{1}, \ldots, y_{s}(s \in \mathbb{N})$ and of complex order $\mu$, let

$$
\Lambda_{\mu, n}\left(y_{1}, \ldots, y_{s} ; z\right):=\sum_{k=0}^{\infty} a_{k} \Omega_{\mu+n k}\left(y_{1}, \ldots, y_{s}\right) z^{k}, \quad\left(a_{k} \neq 0, n \in \mathbb{N}\right)
$$

and

$$
{ }_{q} \Theta_{l, p}^{\mu, n}\left(x_{1}, \ldots, x_{r} ; y_{1}, \ldots, y_{s} ; z\right):=\sum_{k=0}^{[l / p]} a_{k} u_{l-p k, q}^{\left(\alpha_{1}, \ldots, \alpha_{r}\right)}\left(x_{1}, \ldots, x_{r}\right) \Omega_{\mu+n k}\left(y_{1}, \ldots, y_{s}\right) z^{k}
$$

where $[\lambda]$ denotes the integer part of $\lambda \in \mathbb{R}$. Then we have

(3.2) $\sum_{l=0}^{\infty}{ }_{q} \Theta_{l, p}^{\mu, n}\left(x_{1}, \ldots, x_{r} ; y_{1}, \ldots, y_{s} ; \frac{\eta}{t^{p}}\right) t^{l}=\prod_{j=1}^{r} \frac{1}{\left(x_{j} t^{m_{j}} ; q\right)_{\alpha_{j}}} \Lambda_{\mu, n}\left(y_{1}, \ldots, y_{s} ; \eta\right)$

provided that each member of (3.2) exists.

Proof. Let $S:=\sum_{l=0}^{\infty} \Theta_{l, p}^{\mu, n}\left(x_{1}, \ldots, x_{r} ; y_{1}, \ldots, y_{s} ; \frac{\eta}{t^{p}}\right) t^{l}$. Taking $z \rightarrow \frac{\eta}{t^{p}}$ in (3.1), multiplying by $t^{l}$, and summing from $l=0$ to $\infty$ we have

$$
S=\sum_{l=0}^{\infty} \sum_{k=0}^{[l / p]} a_{k} u_{l-p k, q}^{\left(\alpha_{1}, \ldots, \alpha_{r}\right)}\left(x_{1}, \ldots, x_{r}\right) \Omega_{\mu+n k}\left(y_{1}, \ldots, y_{s}\right) \frac{\eta^{k}}{t^{p k}} t^{l}
$$

So the proof is completed by replacing $l$ with $l+p k$ in (3.3).

Lemma 3.2. For the multivariable q-polynomials we have the following addition formula

$$
u_{n, q}^{\left(\alpha_{1}+\beta_{1}, \ldots, \alpha_{r}+\beta_{r}\right)}\left(x_{1}, \ldots, x_{r}\right)=\sum_{k=0}^{n} u_{n-k, q}^{\left(\alpha_{1}, \ldots, \alpha_{r}\right)}\left(x_{1}, \ldots, x_{r}\right) u_{k, q}^{\left(\beta_{1}, \ldots, \beta_{r}\right)}\left(x_{1} q^{\alpha_{1}}, \ldots, x_{r} q^{\alpha_{r}}\right) .
$$


Proof. Take $\alpha_{i} \rightarrow \alpha_{i}+\beta_{i}$ in (2.1).

Now using Lemma 3.2 we immediately get the following result.

Theorem 3.3. For a non-vanishing function $\Omega_{\mu}\left(y_{1}, \ldots, y_{s}\right)$ of complex variables $y_{1}, \ldots, y_{s}(s \in \mathbb{N})$ and for $p \in \mathbb{N}, \alpha=\left(\alpha_{1}, \ldots, \alpha_{r}\right), \beta=\left(\beta_{1}, \ldots, \beta_{r}\right)$ let

$$
\begin{aligned}
& \Lambda_{\mu, \alpha, \beta}^{p, n}\left(x_{1}, \ldots, x_{r} ; y_{1}, \ldots, y_{s} ; z\right):=\sum_{k=0}^{[n / p]} a_{k} u_{n-p k, q}^{\left(\alpha_{1}+\beta_{1}, \ldots, \alpha_{r}+\beta_{r}\right)}\left(x_{1}, \ldots, x_{r}\right) \\
& \times \Omega_{\mu}\left(y_{1}, \ldots, y_{s}\right) z^{k},
\end{aligned}
$$

where $a_{k} \neq 0, n \in \mathbb{N}$. Then we have

$$
\begin{aligned}
& \sum_{k=0}^{n} \sum_{l=0}^{[k / p]} a_{l} u_{n-k, q}^{\left(\alpha_{1}, \ldots, \alpha_{r}\right)}\left(x_{1}, \ldots, x_{r}\right) u_{k-p l, q}^{\left(\beta_{1}, \ldots, \beta_{r}\right)}\left(x_{1} q^{\alpha_{1}}, \ldots, x_{r} q^{\alpha_{r}}\right) \\
& \quad \Omega_{\mu}\left(y_{1}, \ldots, y_{s}\right) z^{l}=\Lambda_{\mu, \alpha, \beta}^{p, n}\left(x_{1}, \ldots, x_{r} ; y_{1}, \ldots, y_{s} ; z\right)
\end{aligned}
$$

provided that each member of (3.5) exists.

Choosing $m_{j}=1(j=1, \ldots, r)$ in $(2.1)$, we obtain a basic (or $q-$ ) analogue of the Chan-Chyan-Srivastava multivariable polynomials $g_{n, q}^{\left(\alpha_{1}, \ldots, \alpha_{r}\right)}\left(x_{1}, \ldots, x_{r}\right)$ generated by (see [1])

$$
\prod_{i=1}^{r} \frac{1}{\left(x_{i} t ; q\right)_{\alpha_{i}}}=\sum_{n=0}^{\infty} g_{n, q}^{\left(\alpha_{1}, \ldots, \alpha_{r}\right)}\left(x_{1}, \ldots, x_{r}\right) t^{n},
$$

where $|t|<\min \left\{\left|x_{1}\right|^{-1}, \ldots,\left|x_{r}\right|^{-1}\right\}$.

If we set $m_{j}=j(j=1, \ldots, r)$ in $(2.1)$, we get a basic (or $\left.q-\right)$ analogue of the multivariable Lagrange-Hermite polynomials $h_{n, q}^{\left(\alpha_{1}, \ldots, \alpha_{r}\right)}\left(x_{1}, \ldots, x_{r}\right)$, generated by (see [2])

$$
\prod_{j=1}^{r} \frac{1}{\left(x_{j} t^{j} ; q\right)_{\alpha_{j}}}=\sum_{n=0}^{\infty} h_{n, q}^{\left(\alpha_{1}, \ldots, \alpha_{r}\right)}\left(x_{1}, \ldots, x_{r}\right) t^{n},
$$

where $|t|<\min \left\{\left|x_{1}\right|^{-1},\left|x_{2}\right|^{-1 / 2}, \ldots,\left|x_{r}\right|^{-1 / r}\right\}$.

Finally we should note that, for every suitable choice of the coefficients $a_{k}(k \in$ $\left.\mathbb{N}_{0}\right)$, if the multivariable function $\Omega_{\mu}\left(y_{1}, \ldots, y_{s}\right),(s=2,3, \ldots)$ is expressed as an appropriate product of several simpler functions, the assertion of the above theorems can be applied in order to derive various families of multilinear and multilateral generating functions for the basic (or $q-$ ) analogue of the generalized multivariable polynomials defined by (2.3). 


\section{REFERENCES}

1. A. Altın, E. Erkuş and F. Taşdelen, The $q$-Lagrange polynomials in several variables, Taiwanese J. Math., 10 (2006), 1131-1137.

2. E. Erkuş, The multivariable $q$-Lagrange-Hermite polynomials, Internat. J. Comput. Numer. Anal. Appl., 6 (2004), 143-151.

3. E. Erkuş and H. M. Srivastava, A unified presentation of some families of multivariable polynomials, Integral Transform. Spec. Funct., 17 (2006), 267-273.

4. V. K. Jain and H. M. Srivastava, Some families of multilinear $q-$ generating functions and combinatorial q-series identities, J. Math. Anal. Appl., 192 (1995), 413-438.

5. E. D. Rainville, Special Functions, The Macmillan Company, New York, 1960.

6. L. J. Slater, Generalized Hypergeometric Functions, Cambridge Univ. Press, London/New York, 1966.

7. H. M. Srivastava and H. L. Manocha, A Treatise on Generating Functions, Halsted Press (Ellis Horwood Limited, Chichester), John Wiley and Sons, New York, 1984.

Esra Erkuş-Duman

Department of Mathematics,

Faculty of Arts and Sciences,

Gazi University,

Teknikokullar 06500, Ankara,

Turkey

E-mail: eduman@gazi.edu.tr 